\title{
Handling of the generation of primary events in Gauss, the LHCb simulation framework
}

\author{
I. Belyaev*†, T. Brambach ${ }^{\ddagger}$, N.H. Brook ${ }^{\S}$, N. Gauvin $₫$, G. Cortill, K. Harrison**, P.F. Harrison ${ }^{\dagger \dagger}$, J. He ${ }^{\ddagger \ddagger}$, \\ P.H. Ilten ${ }^{x}$, C.R. Jones** ${ }^{*}$, M. Lieng ${ }^{\ddagger}$, G. Manca ${ }^{\mathrm{xi}} \|$, S. Miglioranzi ${ }^{\|}$, P. Robbe ${ }^{\ddagger \ddagger} \|$, V. Vagnoni ${ }^{\mathrm{xii}}$, M. Whitehead ${ }^{\dagger \dagger}$ \\ and J. Wishahi ${ }^{\ddagger}$. \\ ${ }^{*}$ Nikhef National Institute for Subatomic Physics, Amsterdam, Netherlands \\ ${ }^{\dagger}$ Institute of Theoretical and Experimental Physics (ITEP), Moscow, Russia \\ ${ }^{\ddagger}$ Fakultät Physik, Technische Universität Dortmund, Dortmund, Germany \\ ${ }^{\S}$ H.H. Wills Physics Laboratory, University of Bristol, Bristol, United Kingdom \\ IEcole Polytechnique Fédérale de Lausanne (EPFL), Lausanne, Switzerland \\ "European Organization for Nuclear Research (CERN), Geneva, Switzerland \\ ${ }^{* *}$ Cavendish Laboratory, University of Cambridge, Cambridge, United Kingdom \\ ${ }^{\dagger}$ Department of Physics, University of Warwick, Coventry, United Kingdom \\ $\ddagger$ LAL, Université Paris-Sud, CNRS/IN2P3, Orsay, France \\ ${ }^{\mathrm{x}}$ School of Physics, University College Dublin, Dublin, Ireland \\ ${ }^{x i}$ Sezione INFN di Cagliari and Università di Cagliari, Cagliari, Italy \\ ${ }^{x i i}$ Sezione INFN di Bologna, Bologna, Italy
}

\begin{abstract}
The LHCb simulation application, Gauss, consists of two independent phases, the generation of the primary event and the tracking of particles produced in the experimental setup. For the LHCb experimental program it is particularly important to model $B$ meson decays: the EvtGen code developed in CLEO and BABAR has been chosen and customized for non-coherent $B$ production as occuring in $p p$ collisions at the LHC. The initial proton-proton collision is provided by a different generator engine, currently PYTHIA 6 for massive production of signal and generic $p p$ collisions events. Beam gas events, background events originating from proton halo, cosmics and calibration events for different detectors can be generated in addition to $p p$ collisions. Different generator packages as available in the physics community or specifically developed in $\mathrm{LHCb}$ are used for the different purposes. Running conditions affecting the events generated such as the size of the luminous region, the number of collisions occuring in a bunch crossing and the number of spill-over events from neighbouring bunches are modeled via dedicated algorithms appropriately configured. The design of the generator phase of Gauss will be described: a modular structure with well defined interfaces specific to the various tasks, e.g. $p p$ collisions, particles' decays, selections, etc. has been chosen. Different implementations are available for the various tasks allowing selecting and combining them as most appropriate at run time as in the case of PYTHIA 6 for $p p$ collisions or HIJING for beam gas. The advantages of such structure, allowing for example to adopt transparently new generators packages will be discussed.
\end{abstract}

\section{INTRODUCTION}

$\mathbf{T}$ HE LHCb [1] simulation application, Gauss [2], [3], consists of two independent phases:

1) the generation of the primary event,

2) the tracking of the particles produced in the experimental setup (detector).

On behalf of the LHCb Collaboration.
The first step can be further divided into two important main parts, both using external generator packages. First, the production of particles coming out of the primary $p p$ collision of the LHC beams. This is usually realized by the PYTHIA [4] general purpose event generator. Second, the decay and time evolution of the produced particles. Mainly EvtGen [5] is used to perform this step. The simulation software application, Gauss, is interfaced to these two external generators, and provides the necessary algorithms to steer the execution of the different generation sequences, and to ensure the coherence between them.

A very generic framework has been designed. The resulting application is flexible enough to be able to generate a very large variety of event types, from beam gas events up to very rare decays of $B$ mesons. Thus different generator packages available in the high-energy physics community have been interfaced to Gauss.

\section{General Software Structure}

The structure of the $\mathrm{LHCb}$ simulation software is represented in Fig. 1, where the two main phases are represented: event generation and detector simulation. The simulation part is based on the GEANT4 package [6] and is described in detail in [3]. The generation step deals with the generation of physics events and uses several generic tools to perform the necessary actions.

\section{A. Main generation algorithm}

The generation of the events is realized in one Gaudi algorithm [7], [8] which implements three main actions: 


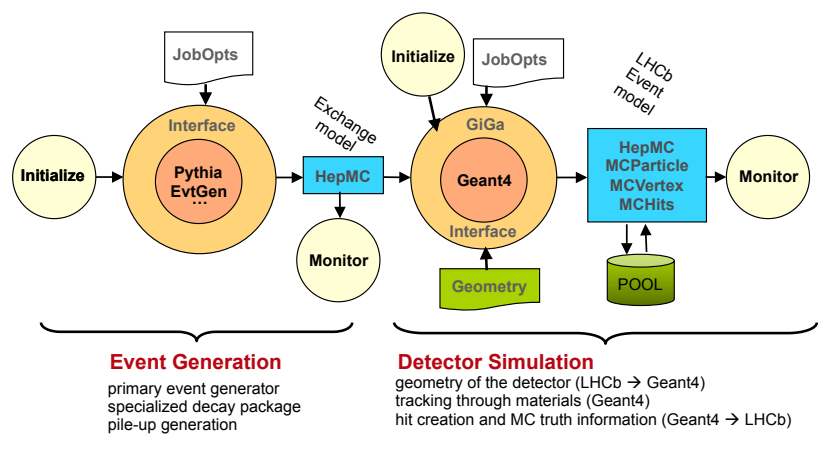

Fig. 1. Structure of the Gauss software

1) Initialization: This step mainly deals with the configuration of the algorithm which is obtained from "configurables" [8], i.e. special python classes built from the $\mathrm{C}++$ components (Services, Algorthms, Tools) which compute from user inputs the value of the parameters to be used for the generation. These values can be for example the energy of the proton beam to consider for the generation, since the LHC can operate at various center-of-mass energies (for example $900 \mathrm{GeV}, 7 \mathrm{TeV}$ or $14 \mathrm{TeV}$ ), or the crossing angle between the beams.

2) Event loop execution: The result of this step is the generation of one physics event corresponding to the criteria defined at the initialization (for example events containing a $b$ quark). The generated event is stored in HepMC format [9] and is then transferred to the simulation step, as illustrated in Fig. 1. It can optionally be saved on a POOL output file [10] to be analyzed in detail later.

In order to realize all computations needed for the generation of one event, several tools are called by the execute method of the algorithm responsible for the generation of physics events. Each tool has a generic interface and several concrete implementations, which are chosen depending on the configuration of the algorithm. This choice of generic tools allows to substitute easily inside the algorithm a method by another one. For example, the generation of beam parameters is usually describing the head-on collision of two proton beams but can be substituted by the description of the collision of one proton beam with one gas molecule in order to simulate beam-gas events. All available tools will be decribed in detail in II-B.

3) Finalization: Monitoring counters are printed at this stage. They allow to count the efficiency of the various generator-level selections applied during the event generation, and to know the cross-sections of the generated processes. These counters are available from the log files of the production simulation jobs. A script extracts from these log files the interesting information and computes numerical results which are available in web pages for the physicists analysing the simulated data.

The generation algorithm also accesses generic LHCb common services. A random generator service provides all tools and algorithms with a common random number generator, including external libraries which are interfaced in such a way that their internal random number generator is replaced by the one of the service. In order to ensure that any event in a sequence of events can be reproduced without generating all preceding events, the random generator seed is set before generating every new event. The seed is set according to a unique combination of integers (run and event numbers) which identify uniquely each event.

A particle property service is used to define properties of particles (mass, lifetime, spin, charge and width). This ensures that all LHCb software (including also the reconstruction and the analysis applications) use the same particle properties. The source of data for the particle properties is the review of particle physics of the Particle Data Group (PDG) [11].

\section{B. Tools for the generation algorithm}

The algorithm described in Sec. II-A calls various tools to realize specific computations.

1) Production Tool: It takes care of the primary $p p$ collision generation: hard process, evolution of the partons up to the formation of hadrons, generation of multiple parton-parton interactions. This is usually realized calling an external generator, such as the general purpose event generators PYTHIA 6 [4], Pythia 8 [12], HERWIG [13], Herwig++ [14] or SHERPA [15]. In this case, the tool is an interface to the generator (which can be either written in FORTRAN or in $\mathrm{C}++$ language). The tool also provides the possibility to configure the external generator in various ways, transferring the configuration commands given by the Gauss user through configurable settings of the tools into the format needed by the external generator. The main external generator used is up to now Pythia 6, whose interface in Gauss will be detailed in Sec. III.

Other implementations of the "Production Tool" are provided in Gauss for specific purposes: an interface to BCVEGPY [16] for the production of the $B_{c}$ meson and an interface to HIJING [17] for the simulation of beam-gas events for example. The possibility to use text files as production engines is also provided, either containing fully generated events, or containing parton level events to be hadronized by another generator. The supported file format are the HepMC ASCII file format or PYTHIA Les Houches Event (LHE) files [18].

2) Decay Tool: It is used to decay hadrons produced by the "Production Tool". The primary event generation stops after the hadronization, and then delegates to the decay tool the generation of decays. Because the LHCb experiment focuses on flavour physics and in particular $B$ physics, it needs a very detailed simulation of $B$ decays, taking into account $C P$ violation effects or angular correlations in decay chains. The EvtGen generator [5] is very well suited for this purpose since it was developed at CLEO and BABAR, experiments also devoted to $B$ physics. The use of EvtGen in $\mathrm{LHCb}$ will be described in detail in Sec. IV.

The SHERPA generator is also interfaced to Gauss as a "Decay Tool" since it also provides the possibility to implement detailed description of decay properties. One important task of the "Decay Tool" is to generate signal decay, i.e. to force the "signal" particle to decay into the decay mode of interest. This allows Gauss users to obtain samples containing 
only the decay mode under study to be able to determine their reconstruction efficiencies or the biases introduced by the experimental setup.

3) Sample Generation Tool: Several categories of event samples are of interest for the $\mathrm{LHCb}$ physics program:

Minimum Bias All events generated by the production generator, with no requirement about their content.

Inclusive Events containing a particle out of a configurable list of particle types. This category is mainly composed of inclusive $c$ and $b$ events, defined as events containing at least one charm hadron or one beauty hadron respectively.

Signal All events containing a particle of a given type (the "signal"), like $B_{s}^{0} /{\overline{B_{s}}}^{0}$ for example. In each event, one of the "signal" particle is forced to decay by the decay generator to a predefined decay mode, such as $B_{s}^{0} \rightarrow J / \psi \phi$. In order to speedup the generation of $B$ events for relatively rare signal hadrons $\left(B_{s}^{0}\right.$ or $\Lambda_{b}$ compared to $B^{+}$and $B^{0}$ for example), the following method is applied: once an event with a $b$ quark is obtained, the same event is re-hadronized several times until the correct type of $B$ meson is found. This is implemented only when using the PYTHIA 6 production generator, and will be explained in detail in Sec. III-C.

Special events Events defined with special generator settings, usually for processes with very low cross-sections, such as $Z^{0}$ production.

Since Inclusive and Signal events have a rather large crosssection, they are then extracted from Minimum Bias and don't need extra special settings of the production generator.

4) Pile-Up Tool: More than one interaction can occur in a given bunch crossing in the experiment, hence in one individual simulated event. Additional interactions with respect to the interaction containing the process of interest are called "Pile-Up Interactions". They are generated by Gauss adding Minimum Bias interactions generated by the production generator on top of the main interaction.

The "Pile-Up Tool" is used to provide the computation of the number of interactions in one event, $N_{\text {int }}$. The default and main usage is to generate $N_{\text {int }}$ following a Poisson law with mean value $\nu$ depending on the total cross-section of the $p p$ collision, $\sigma_{\text {tot }}$, the instantaneous luminosity, $\mathcal{L}$ and the collision frequency of the LHC bunches, $f . \nu$ is determined as:

$$
\nu=\frac{\mathcal{L} \sigma_{\text {tot }}}{f}
$$

For the generation of rare processes, since they have very low cross-sections and cannot be extracted out of Minimum Bias, a different scheme has to be adopted to generate directly the correct $N_{\text {int }}$ distribution. In this case, events containing one interaction with this rare process have $N_{\text {int }}-1$ following a
Poisson distribution with the same mean value $\nu$ as described in (1). The mean number of interactions per event containing the rare process is then $\left\langle N_{\text {int }}\right\rangle=\nu+1$. The possibility to generate single interaction events, for beam-gas generation for example, is also available.

5) Beam Tool: It generates the kinematics of the LHC proton beams. The main usage is to describe two colliding proton beams, with a crossing angle. The crossing angle is smeared by a Gaussian function with $\sigma$ equal to:

$$
\sigma=\sqrt{\frac{\epsilon}{\beta^{*}}}
$$

where $\epsilon$ is the beam emittance and $\beta^{*}$ is the beam $\beta$-function at the collision point. An implementation of the "Beam Tool" also provides the possibility to generate a single beam against a fixed target. This is used for the simulation of beam-gas events, where the fixed target is a molecule of the residual gas in the vacuum pipe of the experiment.

6) Cut Tool: The full simulation of the interactions of the generated events in the $\mathrm{LHCb}$ detector is very time consuming. In order to reject as early as possible in the simulation process events which would anyway be rejected by the analysis, selection cuts at the generator level are implemented. The most simple cut applied to events is to require that the particles of interest travel in the direction of the $\mathrm{LHCb}$ detector, which has a limited acceptance, i.e. that particles have an angle with respect to the beam direction less than $400 \mathrm{mrad}$, with $p_{z}>0$. A more efficient cut that can be applied on "signal" particles is to require that all stable decay products not coming from a long lived particle such as $\Lambda$ have an angle $\theta$ with respect to the beam axis $10<\theta<400 \mathrm{mrad}$ for charged particles and $5<\theta<400 \mathrm{mrad}$ for neutral particles. Many other different implementations are available or can be added easily. Kinematic properties of all the particles generated in the event can be used to apply generator level cuts in the Gauss generation phase.

7) Vertex Smearing Tool: It implements the generation of the luminous region of the LHC collisions at the LHCb Interaction Point. The position of the interaction point is smeared around the mean collision point by Gaussian distributions in $x, y$ and $z$. For the generation of beam-gas events, only the $x$ and $y$ positions are smeared while the $z$ coordinate has a flat distribution.

\section{Sequencing of the generation algorithm}

The actions done by each tool are steered from the main generation algorithm. Figure 2 represents the order in which tools are called, and shows schematically what they realize.

In more detail, the logic of "Signal" sample generation to obtain one event is the following:

1) Compute the number $N_{\text {int }}$ of pile-up interactions for this event, calling the "Pile-Up Tool".

2) Produce $N_{\text {int }}$ interactions of type "Minimum Bias" with the "Production Tool". For each interaction, the beam parameters (4-momentum) are obtained from the "Beam Tool'.

a) Decay all hadrons produced with the "Decay Tool". 


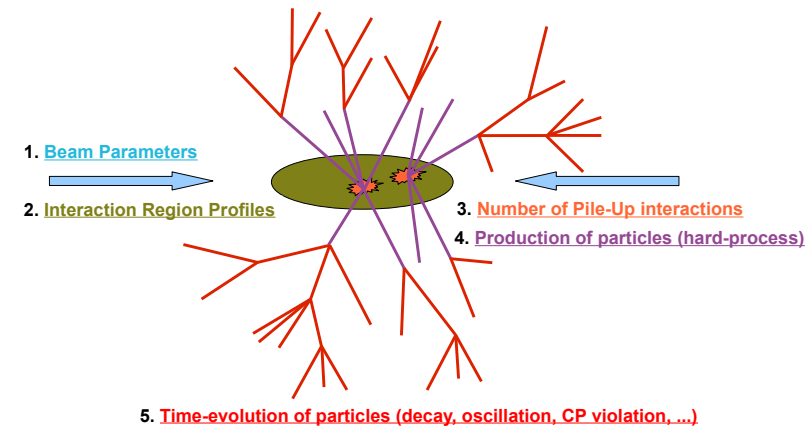

Fig. 2. Sequence of actions for the generation of events

b) Check if one of the interactions contains the signal hadron. If not, go back to 2). This step is arranged by the "Sample Generation Tool".

c) Force the signal particle to decay to the "signal" requested final state, also using the "Decay Tool".

d) Check if the signal particle meets the requirements of the generator level selection, calling the " $\mathrm{Cut}$ Tool". If not, go back to 2).

e) Determine the spatial position of each $N_{\text {int }}$ interactions with the "Vertex Smearing Tool".

The generation of other types of events (Minimum Bias, Inclusive or Special samples) are simplified adaptations of the scheme described above.

\section{USE OF PYThia 6 IN LHCB}

The main production generator used in LHCb is PYTHIA, version 6.4. It is a general purpose event generator widely used in the high-energy physics community and written in FORTRAN. Thanks to a very large number of steering parameters, Pythia can be configured in many different ways. This section describes the settings chosen for the LHCb generation, listed in Tab. I and the interface of Gauss to PYTHIA 6.

\section{A. Physics processes}

The $\mathrm{LHCb}$ experiment is primarily dedicated to the study of rare $D$ and $B$ decays. These hadrons are produced at the

TABLE I

LHCB PYTHIA 6 PARAMETERS

\begin{tabular}{c|c||c|c}
\hline Parameter & Value & Parameter & Value \\
\hline \hline CKIN (41) & 3.0 & PARP (86) & 0.66 \\
MSTP (2) & 2 & PARP (89) & 14000 \\
MSTP (33) & 3 & PARP (90) & 0.238 \\
MSTP (81) & 21 & PARP (91) & 1.0 \\
MSTP (82) & 3 & PARP (149) & 0.02 \\
MSTP (52) & 2 & PARP (150) & 0.085 \\
MSTP (51) & 10042 & PARJ (11) & 0.5 \\
MSTP (142) & 2 & PARJ (12) & 0.4 \\
PARP (67) & 1 & PARJ (13) & 0.79 \\
PARP (82) & 4.28 & PARJ (14) & 0.0 \\
PARP (85) & 0.33 & PARJ (15) & 0.018 \\
MSTJ (26) & 0 & PARJ (16) & 0.054 \\
PARJ (33) & 0.4 & PARJ (17) & 0.131 \\
\hline
\end{tabular}

Leading Order (tree-level) in $2 \rightarrow 2$ QCD processes by the creation of $c \bar{c}$ or $b \bar{b}$ pairs from interactions between light quarks and gluons of the protons. These processes are the pair creation processes whose diagrams are shown in Fig. 3.

$B$ and $D$ production can also emerge from $b$ and $c$ quarks being picked up in the parton distributions of the proton (flavour excitation). They can also be produced by gluon splitting in initial or final state gluon emission. These two processes, represented in Fig. 4, are not negligible at LHC energies even though they are of higher orders. They have to be taken into account for a correct generation of heavy flavours. This is why additional $2 \rightarrow 2$ QCD processes are activated in Gauss, as can be seen in Tab. II which lists the physics processes activated to define "Minimum Bias" events.

TABLE II

LHCb Pythia 6 Minimum Bias PROCESSES

\begin{tabular}{c|c}
\hline Process Number & Descripton \\
\hline \hline 11 & $f+f^{\prime} \rightarrow f+f^{\prime}(\mathrm{QCD})$ \\
12 & $f+\bar{f} \rightarrow f^{\prime}+\overline{f^{\prime}}$ \\
13 & $f+\bar{f} \rightarrow g+g$ \\
28 & $f+g \rightarrow f+g$ \\
53 & $g+g \rightarrow f+\bar{f}$ \\
68 & $g+g \rightarrow g+g$ \\
91 & Elastic scattering \\
92 & Single diffractive $(A B \rightarrow X B)$ \\
93 & Single diffractive $(A B \rightarrow A X)$ \\
94 & Double diffractive \\
95 & Low- $p_{\mathrm{T}}$ scattering \\
$421-439$ & Prompt charmonium \\
$461-479$ & Prompt bottomonium \\
\hline
\end{tabular}

In addition to these processes, single diffractive, double diffractive and elastic processes are also added to obtain a realistic description of "Minimum Bias" events. On top of these processes, the production of prompt charmonium and prompt bottomonium states is also activated, since the detailed study of these particles is also part of the LHCb physics program.

Other processes ( $W, Z$ or Higgs production) can be activated for specific studies. Since the cross-section of these processes is very small, they are not part of the "Minimum Bias" definition and a special configuration of the software is needed to obtain them. The Gauss software provides the possibility to change the value of any of the PYTHIA 6 configuration switches, through Python configurable files.

\section{B. PYTHia 6 tuning}

1) Charged track multiplicity: The principle of PYTHIA tuning for the LHCb simulation is to extrapolate the charged tracks multiplicity observed at experiments running at lower energies to those of the LHC [19], [20]. This is mainly

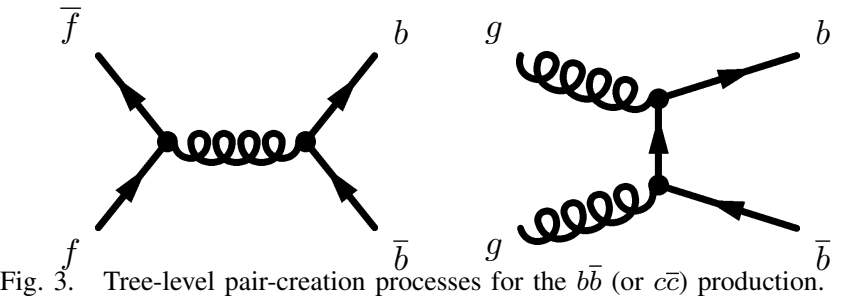



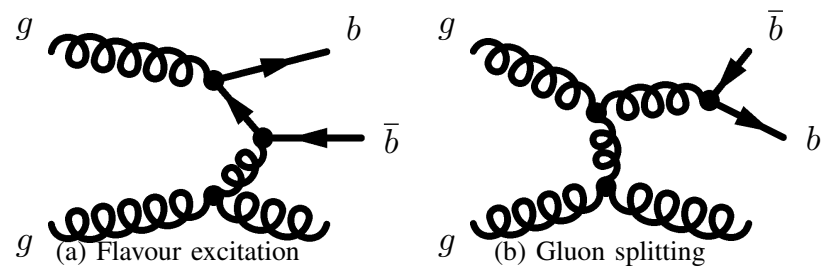

Fig. 4. Higher order processes for the $b \bar{b}$ (or $c \bar{c}$ ) production.

controlled by the $p_{\mathrm{T} \text { min }}$ parameter (PARP (82)) which is the minimal transverse momentum of the parton-parton collision in multiple interactions. The $\mathrm{LHCb}$ simulation software uses the so-called new interleaved multiple interaction model of PYTHIA 6.4 (MSTP $(81)=21)$, with multiple interactions assuming a varying impact parameter and a hadronic matter following a Gaussian matter distribution (MSTP (82) =3).

The mean charged track multiplicity in the central region $\left.\frac{\mathrm{d} n_{\mathrm{ch}}}{\mathrm{d} \eta}\right|_{\eta=0}$ has been measured by the UA5 [22], [23] and CDF [24] experiments at $p p$ or $p \bar{p}$ collisions for various center-ofmass energies, $\sqrt{s}$. Using the LHCb PYTHIA configuration (i.e. with the parameters given in Tab I, in particular using the CTEQ61l Parton Density Function [21]), values of the $p_{\text {T min }}$ parameter are determined to reproduce the measured multiplicities, excluding elastic and single-diffractive events. The evolution of $p_{\mathrm{T} \text { min }}$ as a function of $\sqrt{s}$ is then fitted with the function:

$$
p_{\mathrm{T} \min }=p_{\mathrm{T} \min }^{\mathrm{LHC}}\left(\frac{\sqrt{s}}{\sqrt{s_{0}}}\right)^{2 \epsilon}
$$

where $p_{\mathrm{T} \text { min }}^{\mathrm{LHC}}$ is the extrapolated $p_{\mathrm{T} \text { min }}$ at the reference energy $\sqrt{s_{0}}=14 \mathrm{TeV}$ which is the design energy of the LHC. The result of this fit [25] is shown in Fig. 5 and is used to determine the multiple interaction PYTHIA parameters: $\operatorname{PARP}(82)=p_{\mathrm{T} \text { min }}^{\mathrm{LHC}}=4.28 \mathrm{GeV}$ and $\operatorname{PARP}(90)=2 \epsilon=0.238$.

2) Excited states fraction: A large part of the LHCb physics program is dedicated to $C P$ violation studies. These analyses need $b$ flavour tagging to know the flavour of the studied $B$ hadron at production. Some of the tagging techniques use the sign of the charge of particles produced in excited $B$ decays. It is thus very important to describe precisely the fractions of excited $B^{*(*)}$ produced in $p p$ collisions. These

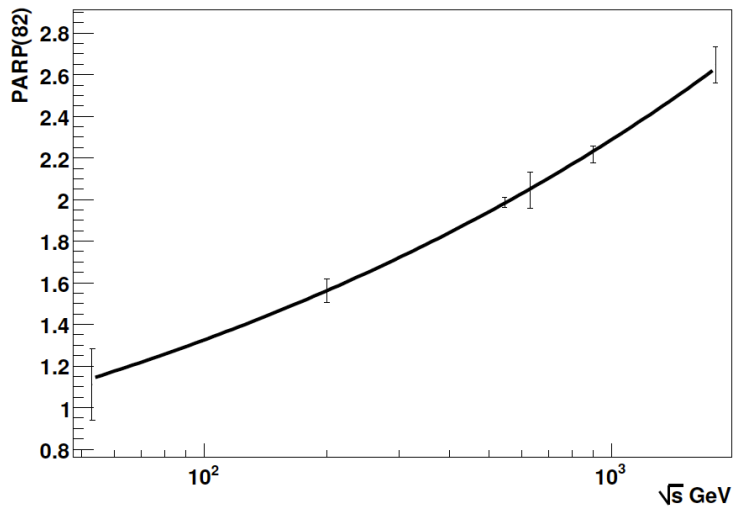

Fig. 5. $\quad p_{\mathrm{T} \min }=\operatorname{PARP}(82)$ as a function of $\sqrt{s}$ fractions are set in order to be compatible with the $B^{*}$ fraction measured at LEP and CDF [26]-[28], to preserve spin counting rules for $B^{* *}$ states and to give $B$ hadronization fractions in agreement with the measurement averages [29], i.e. $f(B)=21 \%, f\left(B^{*}\right)=63 \%$ and $f\left(B^{* *}\right)=16 \%$ for the excited states fractions, and $f_{u}=f_{d}=40.5 \%, f_{s}=10 \%$ and $f_{\text {baryon }}=9 \%$ for the $b$ hadronization fractions into $B^{+}, B^{0}$, $B_{s}^{0}$ and $b$-baryons respectively.

In addition, the PYTHIA parameters governing the production of high spin states are determined to also reproduce the production rates measured at LEP of the following resonances: $\rho(770)^{0}, \omega(782), \phi(1020)$, $K^{*}(892)^{ \pm}$and $D^{*}(2010)^{ \pm}$[30]. This procedure leads to $\operatorname{PARJ}(11)=0.5, \quad \operatorname{PARJ}(12)=0.4, \quad \operatorname{PARJ}(13)=0.79$, $\operatorname{PARJ}(14)=0, \quad \operatorname{PARJ}(15)=0.018, \quad \operatorname{PARJ}(16)=0.054$ and PARJ $(17)=0.131$ [31].

3) Prompt quarkonium spectrum: One other important aspect of the tuning consists in obtaining a realistic spectrum of prompt $J / \psi$ because they are an important source of background in $B$ decays to $J / \psi$ final states which are the golden modes for $C P$ violations studies: $B^{0} \rightarrow J / \psi K_{\mathrm{S}}$ or $B_{s}^{0} \rightarrow J / \psi \phi$. The production models used are the NRQCD models [32], tuned against CDF data [33], [34]. The tuning procedure is described in detail in [35] and is applied directly for the $\mathrm{LHCb}$ simulation.

\section{Repeated hadronization}

In order to have a more efficient generation of $B$ events, a special method called "Repeated Hadronization" has been designed. This method consists in looking for events with $b$ quarks, and then keep the parton level event and repeat the hadronization step until the hadron of interest, or "signal hadron" $\left(B^{0}, B^{+}, B_{s}\right.$ or $\left.\Lambda_{b}\right)$ has been obtained.

In more detail, the sequence of actions performed by this method is:

1) Generate "Minimum Bias" events until one with a $b$ quark has been found, save the parton level event (CALL PYEDIT (21)).

2) Check if the "signal hadron" is present in the event. If yes, go to 4).

3) Repeat the PYTHIA hadronization on the same parton event $(\operatorname{MSTJ}(1)=1$; CALL PYEDIT (22); CALL PYEXEC; MSTJ $(1)=0$ ), decay all excited $B$ hadrons with EvtGen, and go to 2).

4) Check if the "signal hadron" passes the generator level cuts, if not go back to 1). Note that a completely new event has to be regenerated in order to avoid biases.

\section{USE OF EVTGEN IN LHCB}

EvtGen [5] is the main decay engine used in LHCb. It is a package developped in CLEO and BABAR and is particularly interesting for what concerns $B$ decays because it implements a very large number of $B$ decay modes of interest for the $\mathrm{LHCb}$ physics program, and can also take care of angular distribution correlations in complex subsequent decays or time-dependent $C P$ violation in $B$ decays. 


\section{A. Implementation of incoherent $B$ mixing}

EvtGen was however developed for the $\Upsilon(4 S) B$-factories environment and had to be adapted to incoherent $B$ production as seen at the LHC $p p$ collisions. The main difference concerns $B$ mixing. Contrary to $B \bar{B}$ pairs produced at the $\Upsilon(4 S)$, the $B$ mesons from the $b \bar{b}$ pairs produced at hadron collisions evolve independently one from the other. In the EvtGen version adapted by $\mathrm{LHCb}$, a $B$ meson produced as a $B^{0}$ (or $B_{s}^{0}$ ) and evolving into a $\bar{B}^{0}$ (or ${\overline{B_{s}}}^{0}$ ) at the time of its decay, is implemented as a decay $B^{0} \rightarrow \bar{B}^{0}$ (or $\left.B_{s}^{0} \rightarrow{\overline{B_{s}}}^{0}\right)$. The mixing probability is generated for a $B$ initial state as:

$$
P_{\text {mix }}=\frac{x^{2}+y^{2}}{x^{2}+y^{2}+\alpha^{2}\left(2+x^{2}-y^{2}\right)},
$$

and for a $\bar{B}$ initial state:

$$
P_{\text {mix }}=\frac{x^{2}+y^{2}}{x^{2}+y^{2}+\alpha^{-2}\left(2+x^{2}-y^{2}\right)},
$$

where $x=\Delta m, \alpha=\left|\frac{q}{p}\right|$ and $y=\frac{\tau_{\mathrm{H}}-\tau_{\mathrm{L}}}{\tau_{\mathrm{H}}+\tau_{\mathrm{L}}}$.

The mixing parameters $\Delta m$ and $\left|\frac{q}{p}\right|$ are passed to EvtGen through the main decay configuration file [36] with the following syntax: Define qoverp_incohMix_B_s0 1.0, Define dm_incohMix_B_s0 17.8e12, Define qoverp_incohMix_B0 1.0 and Define dm_incohMix_B0 $0.507 e 12$. The lifetime of the heavy and light mass eigenstates, $\tau_{\mathrm{H}}$ and $\tau_{\mathrm{L}}$ respectively, are defined in the EvtGen particle list through four extra particles, $B_{\mathrm{H}}$, $B_{\mathrm{L}}, B_{s \mathrm{H}}$ and $B_{s \mathrm{~L}}$.

Then the lifetime distribution of the neutral $B$ mesons is generated proportional to:

$$
f(t)=e^{-\left(\Gamma_{\mathrm{H}}+\Gamma_{\mathrm{L}}\right) t}\left(\cosh \left(\frac{\left(\Gamma_{\mathrm{L}}-\Gamma_{\mathrm{H}}\right) t}{2}\right)+\cos (\Delta m t)\right)
$$

for unmixed states, and

$$
f(t)=e^{-\left(\Gamma_{\mathrm{H}}+\Gamma_{\mathrm{L}}\right) t}\left(\cosh \left(\frac{\left(\Gamma_{\mathrm{L}}-\Gamma_{\mathrm{H}}\right) t}{2}\right)-\cos (\Delta m t)\right),
$$

for mixed states, where $\Gamma_{\mathrm{H}}=1 / \tau_{\mathrm{H}}$ and $\Gamma_{\mathrm{L}}=1 / \tau_{\mathrm{L}}$.

\section{B. Implementation of CP violation}

Time dependent $C P$ violation implementation in the original EvtGen version is also based on the fact that the $B$ mesons are produced coherently from the $\Upsilon(4 S) \rightarrow B^{0} \bar{B}^{0}$ decay and thus cannot be applied directly to the LHC environment. Correct generation of time dependent $C P$ violation requires also a special interface with the generation steering framework. A consequence of $C P$ violation is that the time-integrated $C P$ asymetry of $B$ decays into $C P$ eigenstates is different from 0 . Since neutral $B$ and $\bar{B}$ mesons are produced in equal amounts by the production generator, PYTHIA (apart from small production asymetries), forcing each $B$ meson to decay to a $C P$ violating decay mode to obtain a "signal" sample will result also in a sample with equal amounts of $B$ and $\bar{B}$ mesons, thus not respecting the correct integrated $C P$ asymmetry.
To overcome this problem, a dedicated interface between EvtGen and Gauss has been designed. The basic principle of this interface is that the flavour at production of the "signal" $B$ hadron is generated by EvtGen, depending on the generated $B$ lifetime, $t$, i.e. EvtGen can request the flavour of the $B$ produced by PYTHIA to be flipped. The $B$ and $\bar{B}$ decay amplitudes are normalized such that they are equal at $t=0$. In the final sample, only events for which the $B$ flavour generated by EvtGen matches the flavour produced by PYTHIA are kept. This allows not only to generate the correct $C P$ integrated asymmetry, but also to keep possible production asymmetries.

\section{Interface with PYTHIA 6}

Particle decays are configured in EvtGen by a decay file which contains a list as complete as possible of possible decay modes for all particles [36]. However, a lot of decay modes have not been measured and the listed decay modes do not saturate the particle decay widths. The decay to these unknown modes is delegated by EvtGen to JetSet in the original version of EvtGen, the constituent quarks of the hadron being hadronized to form the final state. Since in $\mathrm{LHCb}$, PYTHIA 6 is also used as production generator, the original EvtGen interface to JetSet has been replaced by an interface to Pythia 6. Gauss uses a single instance of Pythia 6, shared between the production generator and EvtGen. PYTHIA settings have been chosen not to have any interference between the two usages. In particular, particle properties are set from the Gauss framework in PYTHIA and EvtGen with exactly the same values, to ensure coherence inside the entire generation process.

The interface of EvtGen to PYTHIA 6 mainly consists in transforming the internal EvtGen event records into PYTHIA event record (FORTRAN common blocks), call the necessary PYTHIA routines to handle particle decays, and then retrieve and transfer the result of the decay from PYTHIA event record to the EvtGen format. At initialization, the PYTHIA decay table is updated to take into account the modes requested by EvtGen.

\section{Signal decays}

EvtGen offers the possibility to force decays of particles to predefined "signal" modes, using aliases. This possibility is used in the Gauss software to produce "signal" samples. However, again because of complications due to the hadronic environment, a special interface had to be designed to use this feature correctly. The main reason for that is that when generating "signal" samples, only one of the $B$ hadrons produced in the event has to be forced to decay to the signal mode, and there are at least two $B$ hadrons produced per $b \bar{b}$ event. For the generation of a signal $B^{0}$ sample for example, an event with both a $B^{0}$ and a $\bar{B}^{0}$ will have twice more probability to decay to the signal mode than an event with only one $B^{0}$ (and a $B^{-}$ for example). In order to establish the correct proportion in the generated sample of $B^{0} \bar{B}^{0}$ with respect to $B^{0} \bar{B}_{x}+\bar{B}^{0} B_{x}$, where $B_{x}$ is a $b$-hadron which is not a $B^{0}$, events where only one $B^{0}$ and no $\bar{B}^{0}$ or only one $\bar{B}^{0}$ and no $B^{0}$ are rejected 
with probability $\frac{1-\mathcal{B}}{2-\mathcal{B}}$, where $\mathcal{B}$ is the branching fraction of the signal decay mode.

\section{OTHER GENERATORS}

We already mentioned in Sec. II-B1 that in addition to PYTHIA 6 other generators have been interfaced to Gauss as production engines: they consist of PYTHIA 8 [12], HERWIG [13], Herwig++ [14] and SHERPA [15]. In addition for beam gas interactions a special nucleus-nucleus generator, called HIJING is used: it allows to generate protons impinging on any of the residuals gas atoms present in the vacuum pipe as $\mathrm{H}, \mathrm{C}, \mathrm{O}$. Machine induced background can also be generated via a special in-house generator that samples the results of beam losses transported to the $\mathrm{LHCb}$ cavern. A detailed description of this generator is available in [37]. Finally for special calibration or for beam studies a particle gun algorithm is available with various implementations, for example for optical photons or cosmic rays.

\section{CONCLUSION}

The logic and implementation of the generation of physics events for the LHCb simulation software has been described. It allows to interface different generators available within the high-energy physics community and also provides tools to generate events in conditions as close as possible to the real conditions seen at the $\mathrm{LHCb}$ experimental setup.

\section{ACKNOWLEDGMENTS}

We wish to thank D. Lange and A. Ryd for their help in adapting EvtGen to the $\mathrm{LHCb}$ needs, and for their support when incorporating our modifications in the main EvtGen version.

\section{REFERENCES}

[1] The LHCb Collaboration, A. A. Alves et al., "The LHCb Detector At The LHC", JINST 3 (2008) S08005.

[2] The LHCb Collaboration, R. Antunes Nobrega et al., "LHCb Computing Technical Design Report", CERN-LHCC-2005-019 (2005).

[3] I. Belyaev et al., "Simulation application for the LHCb experiment, In the Proceedings of 2003 Conference for Computing in High-Energy and Nuclear Physics (CHEP 03), La Jolla, California, 24-28 Mar 2003, arXiv:physics/0306035 (2003)

[4] T. Sjostrand, S. Mrenna and P. Z. Skands, "Pythia 6.4 Physics and Manual”, JHEP 0605, 026 (2006)

[5] D. J. Lange, "The EvtGen particle decay simulation package", Nucl. Instrum. Meth. A 462 (2001) 152.

[6] The Geant4 Collaboration, S. Agostinelli et al., "Geant4, a simulation toolkit“, Nucl. Instr. and Meth. Phys. Res. A 179 (2001) 299.

[7] G. Barrand et al., "'GAUDI - A software architecture and framework for building HEP data processing applications", Comput. Phys. Commun. 140 (2001) 45.

[8] M. Clemencic, H. Degaudenzi, P. Mato, S. Binet, W. Lavrijsen, C. Leggett and I. Belyaev, "Recent Developments In The LHCb Software Framework GAUDI", J. Phys. Conf. Ser. 219 (2010) 042006.

[9] M. Dobbs and J. B. Hansen, "The HepMC C++ Monte Carlo event record for High Energy Physics", Comput. Phys. Commun. 134 (2001) 41.

[10] D. Duellmann, M. Frank, G. Govi, I. Papadopoulos and S. Roiser, "The POOL data storage, cache and conversion mechanism", In the Proceedings of 2003 Conference for Computing in High-Energy and Nuclear Physics (CHEP 03), La Jolla, California, 24-28 Mar 2003 [arXiv:physics/0306084].

[11] Particle Data Group, K. Nakamura et al., J. Phys. G 37, 075021 (2010)

[12] T. Sjostrand, S. Mrenna and P. Z. Skands, "A Brief Introduction to Pythia 8.1”, Comput. Phys. Commun. 178 (2008) 852.
[13] G. Corcella et al., "HERWIG 6.5: an event generator for Hadron Emission Reactions With Interfering Gluons (including supersymmetric processes)," JHEP 0101 (2001) 010

[14] M. Bahr et al., "Herwig++ Physics and Manual", Eur. Phys. J. C 58 (2008) 639

[15] T. Gleisberg, S. Hoeche, F. Krauss, M. Schonherr, S. Schumann, F. Siegert and J. Winter, "Event generation with SHERPA 1.1", JHEP 0902 (2009) 007

[16] C. H. Chang, C. Driouichi, P. Eerola and X. G. Wu, "BCVEGPY: An Event generator for hadronic production of the $B_{c}$ meson", Comput. Phys. Commun. 159 (2004) 192.

[17] M. Gyulassy and X. N. Wang, "HIJING 1.0: A Monte Carlo program for parton and particle production in high-energy hadronic and nuclear collisions," Comput. Phys. Commun. 83 (1994) 307.

[18] J. Alwall et al., "A standard format for Les Houches event files", Comput. Phys. Commun. 176 (2007) 300.

[19] P. Bartalini et al., "Tuning of multiple interactions generated by PYTHIA", LHCb-99-028.

[20] N. Brook et al., "Studies on the tuning of Minimum Bias events at LHCb", LHCb-2005-023.

[21] J. Pumplin, D. R. Stump, J. Huston, H. L. Lai, P. M. Nadolsky and W. K. Tung, "New generation of parton distributions with uncertainties from global QCD analysis," JHEP 0207 (2002) 012.

[22] The UA5 Collaboration, G.J. Alner et al., Z. Phys. C33 (1986) 1.

[23] The UA5 Collaboration, K. Alpgøard et al., Phys. Lett. 112B (1982) 193.

[24] The CDF Collaboration, F. Abe et al., Phys. Rev. D41 (1990) 2330.

[25] A. Muir, "Pythia minimum bias Studies and the RICH1 Spherical Mirror for the LHCb Experiment", PhD Thesis, http://amuir.home.cern. ch/amuir/Alex_Muir_thesis.pdf

[26] The ALEPH Collaboration, R. Barate et al., "Resonant structure and flavour tagging in the $B \pi^{ \pm}$system using fully reconstructed $B$ decays", Phys. Lett. B 425 (1998) 215.

[27] The OPAL Collaboration, R. Akers et al., "Observations of $\pi-B$ charge - flavor correlations and resonant $B \pi$ and $B K$ production", Z. Phys. C 66 (1995) 19.

[28] The CDF Collaboration, A. A. Affolder et al., "Observation of orbitally excited $B$ mesons in $p \bar{p}$ collisions at $\sqrt{s}=1.8$ TeV", Phys. Rev. D 64 (2001) 072002.

[29] Heavy Flavour Averaging Group (HFAG), results prepared for the 2010 Review of Particle Physics, http://www.slac.stanford.edu/xorg/hfag/osc/ PDG_2010/

[30] G. D. Lafferty, P. I. Reeves and M. R. Whalley, "A Compilation of inclusive particle production data in $e^{+} e^{-}$annihilation", J. Phys. G 21 (1995) A1.

[31] K. Lessnoff, "A Study of the LHCb Experiments Sensitivity to $C P$ Violation in Mixing and to Production Asymmetry in $B_{s}$ Mesons, Using Semi-Leptonic Decays", PhD Thesis, CERN-THESIS-2010-076.

[32] G. T. Bodwin, E. Braaten and G. P. Lepage, "Rigorous QCD analysis of inclusive annihilation and production of heavy quarkonium", Phys. Rev. D 51 (1995) 1125, [Erratum-ibid. D 55 (1997) 5853].

[33] The CDF Collaboration, D. Acosta et al., "Measurement of the $J / \psi$ meson and $b$-hadron production cross sections in $p \bar{p}$ collisions at $\sqrt{s}=$ 1960 GeV", Phys. Rev. D 71 (2005) 032001.

[34] The CDF Collaboration, D. Acosta et al., " $\Upsilon$ production and polarization in $p \bar{p}$ collisions at $\sqrt{s}=1.8 \mathrm{TeV}$ ", Phys. Rev. Lett. 88 (2002) 161802.

[35] M. Bargiotti and V. Vagnoni, "Heavy quarkonia sector in PYTHIA 6.324: Tuning, validation and perspectives at LHC(b)", CERN-LHCB2007-042 (2007).

[36] http://svnweb.cern.ch/world/wsvn/lhcb/DBASE/trunk/Gen/DecFiles/ dkfiles/DECAY.DEC

[37] R. B. Appleby, H. Burkhardt, G. Corti, Y. I. Levinsen, M .H. Lieng and $\mathrm{V}$.Talanov, "Simulation of Machine Induced Background in the $\mathrm{LHCb}$ Experiment: Methodology and Implementation", in these proceedings. 